right ventricular outflow tract as an alternative permanent pacing site: long-term follow-up. PACE 1991;14:3-6.

45. Scott S, Accorti P, Callaghan F, Abels D, Boveja B. Ventricular and atrial defibrillation using new transvenous tripolar and bipolar leads with 5 French electrodes and 8 French subcutaneous catheters. PACE 1991;14:1893-8.

46. Davy JM, Fain ES, Dorian P, Winkle RA. The relationship between successful defibrillation and delivered energy in open-chest dogs: reappraisal of the "defibrillation threshold" concept. AM HEART J 1987;113:77-84.

47. Jones DL, Irish WD, Klein GJ. Defibrillation efficacy: com- parison of defibrillation threshold versus dose-response curve determination. Circ Res 1991;69:45-51.

48. Bach Jr SM, Barstad J, Harper N, Mayer D, Moser S, Smutk: M, Theis R, Wollins J. Initial clinical experience: Endotak-implantahle transvenous defibrillator system [Abstract]. . I Am Coll Cardiol 1989;13:65A.

49. An H, Saksena S, Mehra R, Tullo N, Krol R, Smits K. Effect of right ventricular cathode configuration on endocardial cardioversion and defibrillation with dual electrode systems and monophasic shocks [Abstract]. PACE 1990;13:511.

\title{
Outcome of endocardial resection in 33 patients with coronary artery disease: Correlation with ventricular tachycardia morphology
}

The results in 33 patients with ventricular tachycardia (VT) treated by endocardial resection were reviewed, with special emphasis on the presence of single or multiple morphologies preoperatively and intraoperatively. Multiple VT morphologies were induced in 16 patients and a single VT morphology was induced in the remaining 17. Intraoperative programmed stimulation failed to induce VT in eight patients and visually-directed endocardial resection was performed. The remaining patients underwent map-guided resection. The surgical success rate did not correlate with any morphologic characteristics of the VT, such as bundle branch block pattern or axis. In addition, concordance of VT morphologies preoperatively and intraoperatively before resection did not correlate with the surgical success rate. However, patients in whom multiple morphologies of VT were induced intraoperatively had a significantly higher success rate (100\%) compared with those patients in whom only a single morphology was induced intraoperatively $(50 \%, p<0.05)$. Long-term follow-up was maintained in 26 patients. Ventricular tachycardia recurred in two patients and VF recurred in two others who did not have inducible VT 1 week after endocardial resection. In conclusion, neither the preoperative morphologic characteristics of VT nor discordance between the morphologies of VT induced preoperatively and in the operating room influenced the outcome of endocardial resection. However, the surgical success rate is higher when multiple morphologies of VT are inducible in the operating room than when only one VT morphology is inducible. (AM HEART J 1992;124:1500.)

Mark J. Niebauer, MD, Marvin Kirsh, MD, Alan Kadish, MD, Hugh Calkins, MD, and Fred Morady, MD Ann Arbor, Mich.

From the Division of Cardiology, Department of Internal Medicine and Division of Thoracic Surgery, Department of General Surgery, University of Michigan Medical Center.

Received for publication May 15, 1992; accepted July 15, 1992.

Reprint requests: Fred Morady, MD, University of Michigan Medical Center, 1500 East Medical Center Drive, B1 F245, Ann Arbor, MI 48109-0022. $4 / 1 / 41314$
Surgical treatment of intractable ventricular tachycardia (VT) has been performed, with various modifications in technique, since $1959 .{ }^{1}$ One approach involves endocardial resection (ER) guided by intraoperative mapping. ${ }^{2}{ }^{3}$ The presence of multiple morphologic forms of VT has been associated with inef- 
ficacy of ER. ${ }^{4}$ However, the concordance between the preoperative and intraoperative $\mathrm{VT}$ morphologies to date has not been examined in detail. In this retrospective study, the results of ER in 33 patients are reported, with particular attention to the preoperative and intraoperative morphologies of V'T induced by programmed stimulation.

\section{METHODS}

Patient characteristics. Thirty-three patients (26 men and 7 women) underwent ER between 1984 and 1990 at the University of Michigan and Ann Arbor Veterans Administration Medical Centers. The patients ranged in age from 28 to 74 years (mean \pm standard deviation, $58 \pm 12$ ) at the time of surgery. Each patient had coronary artery disease and a history of myocardial infarction, and was referred for management of sustained monomorphic VT refractory to pharmacologic therapy with a mean of $2.5 \pm 1.2$ antiarrhythmic agents. The mean left ventricular ejection fraction as determined by contrast ventriculography was $0.30 \pm 0.11$. Each patient had angiographic evidence of an anterior, apical, or inferior wall aneurysm.

Electrophysiologic testing. Electrophysiology tests were performed at least 5 half-lives after antiarrhythmic drug therapy was discontinued and after informed consent was obtained. Each patient underwent programmed ventricular stimulation 1 to 7 days preoperatively, intraoperatively, and 1 week postoperatively. During the preoperative and postoperative electrophysiology tests, three $6 \mathrm{~F}$ quadripolar electrode catheters were inserted into a femoral vein and were positioned in the right atrium, His bundle position, and right ventricle. For VT mapping in the left ventricle, a $6 \mathrm{~F}$ quadripolar electrode catheter was inserted into a femoral or brachial artery and was passed retrogradely across the aortic valve into the left ventricle. Leads $\mathrm{V}_{1}$, I, III, and the intracardiac electrograms were recorded at a paper speed of $100 \mathrm{~mm} / \mathrm{sec}$ on a Siemens-Elema Mingograf -7 recorder (Siemens Medical Instrumentation, Inc., Iselin, N.J.). Programmed stimulation was performed with a programmable stimulator (Bloom Associates, Ltd., Reading, $\mathrm{Pa}$.) using stimuli $2 \mathrm{msec}$ in duration and at least twice the diastolic threshold. To induce VT, one to three ventricular extrastimuli were delivered following eight-beat basic drive trains at cycle lengths of 400 to $600 \mathrm{msec}$, first at the right ventricular apex, then at the outflow tract or septum. Unless the VT required immediate termination, a 12-lead electrocardiogram was recorded. In some cases, more than one morphology of VT was induced in a given patient. Sustained VT was defined as VT lasting longer than 30 seconds and nonsustained VT was defined as VT lasting from 6 to 30 seconds. Whenever possible, the site of origin of the VT was localized by endocardial activation mapping or pace mapping.

Intraoperative mapping and ER. Each patient was taken to the operating room and was anesthetized with fentanyl before the intraoperative tesling. Programmed stimulation was performed after initiation of cardiopulmonary bypass and after ventriculotomy, with the same stimulation protocol used during preoperative testing. When induced, VT was characterized according to its morphology. The patient underwent endocardial activation mapping using a hand-held electrode, and then underwent resection of the reentrant circuit by previously described means. ${ }^{5}$ A cryogenic probe was used in seven patients, alone or as an adjunct to surgical resection, because of the location of the mapped focus or endocardial scar. Once the focus was resected or ablated, programmed stimulation was repeated in an attempt to reinduce VT. This process was repeated until VT was not inducible, or until mapping was no longer possible. When VT was not inducible intraoperatively, a visually-directed $\mathrm{ER}$, in which all identifiable endocardial scar tissue is resected, was performed. ${ }^{6}$ Five patients also underwent coronary artery bypass grafting after ER.

Postoperative testing. Programmed ventricular stimulation was performed in each patient 7 days after the operation, using the same stimulation protocol used in the preoperative test. If VT was induced, it was characterized according to its morphology and cycle length.

Long-term follow-up. Long-term follow-up was maintained in 26 of the 28 patients discharged after the postoperative electrophysiologic (EP) study. Information regarding the patient's health status and details of his or her medical treatment was obtained from either the referring physicians, by review of the patient's medical record, or by direct contact with the patient or his or her immediate family.

Analysis of data. Data were compared using the chi square test or the Student's paired $t$ test. A $p$ value $<0.05$ level was considered statistically significant.

\section{RESULTS}

Short-term results of surgery. Five of the 33 patients $(15 \%)$ died within 1 week of surgery of either sepsis, pneumonia, or shock caused by severe left ventricular dysfunction. These five patients did not survive to undergo the postoperative EP test, and therefore the short-term surgical results could not be determined. The remaining 28 patients underwent a postoperative EP test, and VT could not be induced in 24 $(86 \%)$.

Morphologic characteristics of induced VT. Sustained monomorphic VT was inducible in each of the 33 patients preoperatively, but in only 22 of the 33 patients $(67 \%)$ intraoperatively $(p<0.05)$. The morphologies of V'T induced by programmed stimulation preoperatively, intraoperatively, and postoperatively are described in Table I.

Among the 28 patients who survived the operation, VT induced during the preoperative EP test had a right bundle branch block configuration in 22 patients and a left bundle branch block configuration in 18 patients. The number of VTs exceeds the number of patients because more than one morphology of VT could be induced in 16 patients. 
Table I. Morphologies of ventricular tachycardia induced preoperatively, intraoperatively, and postoperatively

\begin{tabular}{|c|c|c|c|c|}
\hline $\begin{array}{c}\text { Patient } \\
\text { No. }\end{array}$ & Preop & $\begin{array}{c}\text { Intraop } \\
\text { before } S E R\end{array}$ & $\begin{array}{c}\text { Intraop } \\
\text { after } S E R\end{array}$ & Postop \\
\hline \multirow[t]{3}{*}{1} & LBSA & LBSA & None & None \\
\hline & LBIA & RBIA & & \\
\hline & RBIA & & & \\
\hline \multirow[t]{2}{*}{2} & LBSA & LBSA & LBSA & ND \\
\hline & & RBIA & & \\
\hline \multirow[t]{3}{*}{3} & RBSA & RBIA & None & None \\
\hline & & LBSA & & \\
\hline & & IND & & \\
\hline \multirow[t]{2}{*}{4} & RBSA & RBSA & None & None \\
\hline & & LBSA & & \\
\hline \multirow[t]{2}{*}{5} & RBIA & None & None & None \\
\hline & LBSA & & & \\
\hline \multirow[t]{2}{*}{6} & RBIA & RBIA & None & None \\
\hline & RBSA & LBSA & & \\
\hline \multirow[t]{2}{*}{7} & LBSA & None & None & None \\
\hline & RBSA & & & \\
\hline \multirow[t]{2}{*}{8} & LBSA & LBSA & None & None \\
\hline & RBIA & RBIA & & \\
\hline 9 & RBIA & LBSA & LBIA & None \\
\hline \multirow[t]{2}{*}{10} & RBSA & RBSA & None & ND \\
\hline & LBSA & RBIA & & \\
\hline 11 & RBSA & RBSA & RBSA & RBSA \\
\hline 12 & IND & None & None & None \\
\hline \multirow[t]{3}{*}{13} & LBSA & None & None & None \\
\hline & RBIA & & & \\
\hline & LBIA & & & \\
\hline 14 & LBIA & None & None & None \\
\hline 15 & RBSA & LBSA & None & None \\
\hline 16 & LBSA & None & None & LBSA \\
\hline \multirow[t]{2}{*}{17} & LBSA & LBSA & None & None \\
\hline & & RBIA & & \\
\hline \multirow[t]{3}{*}{18} & RBSA & RBIA & None & None \\
\hline & & LBSA & & \\
\hline & & LBIA & & \\
\hline \multirow[t]{2}{*}{19} & RBSA & RBSA & None & None \\
\hline & & LBIA & & \\
\hline \multirow[t]{2}{*}{20} & LBSA & None & None & None \\
\hline & RBSA & & & \\
\hline 21 & RBSA & UNK & LBSA & LBSA \\
\hline \multirow[t]{3}{*}{22} & RBSA & None & None & None \\
\hline & RBSA & & & \\
\hline & RBIA & & & \\
\hline \multirow[t]{3}{*}{23} & RBSA & RBSA & None & None \\
\hline & RBIA & RBIA & & \\
\hline & LBSA & LBSA & & \\
\hline \multirow[t]{2}{*}{24} & RBIA & RBSA & None & None \\
\hline & LBSA & LBIA & & \\
\hline 25 & RBSA & UNK & UNK & ND \\
\hline 26 & LBSA & UNK & None & None \\
\hline & RBIA & & & \\
\hline 27 & LBSA & RBSA & None & None \\
\hline & & IND & & \\
\hline 28 & LBSA & LBSA & RBIA & None \\
\hline 29 & LBSA & IND & None & LBSA \\
\hline & RBSA & & & \\
\hline 30 & UNK & RBIA & IND & ND \\
\hline 31 & LBSA & LBSA & None & None \\
\hline & RBIA & RBSA & & \\
\hline 32 & RBIA & $\mathrm{RB}$ ?A & None & ND \\
\hline
\end{tabular}


Table I. - Continued

\begin{tabular}{clccc}
$\begin{array}{c}\text { Patient } \\
\text { No. }\end{array}$ & Preop & $\begin{array}{c}\text { Intraop } \\
\text { before SER }\end{array}$ & $\begin{array}{c}\text { Intraop } \\
\text { after SER }\end{array}$ & Postop \\
33 & RBSA & & None & None \\
& RBIA & IND & & IND \\
& LBIA & IND & LBSA & \\
\hline
\end{tabular}

LB/RB, Left/right bundle branch morphology; $S A / I A$, superior/inferior axis; $U N K$, unknown; None, no inducible VT; IND, indeterminate morphology; $N D$ not determined because of perioperative death before postoperative electrophysiology test; $S E R$, subendocardial resection.

Table II. Morphology of ventricular tachycardia and outcome of surgery

\begin{tabular}{|c|c|c|c|c|}
\hline \multirow[b]{2}{*}{ Number of VT forms ${ }^{*}$} & \multicolumn{2}{|c|}{ Bundle branch block } & \multicolumn{2}{|c|}{ Axis } \\
\hline & Right & Left & Superior & Inferior \\
\hline Preoperative & 22 & 18 & 26 & 13 \\
\hline Successful outcome & $15(68 \%)$ & $13(72 \%)$ & $19(73 \%)$ & $10(77 \%)$ N.S. \\
\hline Intra-Op A & 14 & 14 & 17 & 10 \\
\hline Successful outcome & $12(86 \%)$ & $13(93 \%)$ & $15(88 \%)$ & $10(100 \%)$ N.S. \\
\hline
\end{tabular}

Intra-Op A, Intraoperative preresection.

*Sum of morphologies exceeds patient numbers because some patients had more than one VT morphology.

Concordance between preoperative and intraoperative VT morphologies was defined as the presence of at least one V'T morphology that was identical during the preoperative and intraoperative EP tests. Concordance was present in 12 of 28 patients $(43 \%)$. Among these patients, the mean cycle lengths of the VTs induced during the two tests were not significantly different $(337 \pm 81$ msec versus $297 \pm 75$ msec, respectively).

Correlation between VT morphology and surgical result. Neither the morphology nor the axis of the VT induced either preoperatively or intraoperatively was associated with a successful outcome of endocardial resection (Table II). The presence of concordance between the VTs induced preoperatively and intraoperatively also did not correlate with the surgical results. VT was no longer inducible 1 week postoperatively in 10 of 11 patients (91\%) who had identical preoperative and intraoperative VT morphologies, compared with five of five patients $(100 \%)$ who had different morphologies preoperatively and intraoperatively $(p>0.05)$.

The presence of multiple VT morphologies induced intraoperatively was associated with a successful outcome of endocardial resection. The outcome was successful in $100 \%$ of the 13 patients in whom more than one VT morphology was induced in the operating room, as compared with three of six $(50 \%)$ patients who exhibited a single VT morphology intraoperatively $(p<0.05)$.

Map-guided versus visually-directed endocardial re- section. Among the 28 patients who survived the operation, the 20 patients in whom VT could be induced intraoperatively underwent map-guided endocardial resection and the other eight patients underwent a visually-directed resection. A similar percentage of these two groups ( $85 \%$ and $88 \%$, respectively) did not have inducible VT 1 week after the operation $(p>0.05)$.

VT morphologies in patients with inducible VT postoperatively. Among the four patients in whom sustained monomorphic VT was inducible 1 week after surgery, the VT morphology was the same as the preoperative VT morphology in three patients and was different in the other patient.

Long-term results. Complete follow-up information was obtained in 26 of the 28 patients who underwent preoperative, intraoperative, and postoperative electrophysiologic testing (Table III). Two of the four patients who still had inducible VT 1 week after surgery were treated with quinidine, while the others received an implantable cardioverter-defibrillator (ICD). During a mean of 49.5 months of follow-up, the two patients treated with quinidine have not had symptomatic VT and one of the patients who underwent implantation of the ICD has experienced discharges from the device and encainide was therefore added to her therapy. She is otherwise without complaints. The other patient who received an ICD died suddenly 3 months later.

Among 24 patients who did not have inducible VT 1 week postoperatively, 14 have remained asymp- 
Table III. Long-term patient follow-up

\begin{tabular}{|c|c|c|c|c|}
\hline Patient No. & $\begin{array}{l}\text { Follow-up } \\
\quad(m o)\end{array}$ & $V T$ & Treatment & Status \\
\hline 1 & 30 & None & & Alive \\
\hline 2 & 0 & - & & Died, postop \\
\hline 3 & - & Unknown & & Unknown \\
\hline 4 & 24 & None & & Alive, Txp \\
\hline 5 & 60 & RBSA & Amiodarone + Mexilitene & Alive \\
\hline 6 & 24 & None & & Alive, Txp \\
\hline 7 & 24 & None & & Alive \\
\hline 8 & 48 & None & & Alive \\
\hline 9 & 24 & None & & Alive \\
\hline 10 & 0 & - & & Died, postop \\
\hline 11 & 84 & RBSA & Quinidine & Alive \\
\hline 12 & 41 & None & & Alive \\
\hline 13 & 24 & None & & Alive \\
\hline 14 & 7 & None & & Died, VF \\
\hline 15 & 74 & None & & Alive \\
\hline 16 & 51 & LBSA & ICD & Alive \\
\hline 17 & - & Unknown & & Unknown \\
\hline 18 & 18 & None & & Died, VF \\
\hline 19 & 1 & None & & Died, CHF \\
\hline 20 & 22 & LBIA & ICD, Encainide & Alive \\
\hline 21 & 3 & LBSA & ICD & Died \\
\hline 22 & 24 & None & & Alive \\
\hline 23 & 39 & None & & Alive \\
\hline 24 & 8 & None & & Died, MI \\
\hline 25 & 0 & - & & Died, postop \\
\hline 26 & 57 & None & & Alive \\
\hline 27 & 36 & None & & Died, CHF \\
\hline 28 & 18 & None & & Alive \\
\hline 29 & 60 & LBSA & Quinidine & Alive \\
\hline 30 & 0 & - & & Died, postop \\
\hline 31 & 33 & None & & Alive \\
\hline 32 & 0 & - & & Died, postop \\
\hline 33 & 6 & None & & Died, septicemia \\
\hline
\end{tabular}

ICD, Implantable cardioverter-defibrillator; $C H F$, congestive heart failure; $M I$, myocardial infarction; $V F$, ventricular fibrillation; $T \times p$, heart transplant; other abbreviations as in Table I.

tomatic and are currently not receiving antiarrhythmic therapy after a mean of 34.6 months. Two of these patients have received heart transplantation 1 and 2 years after ER for end-stage ischemic cardiomyopathy.

Two patients who did not have inducible VT postoperatively experienced episodes of sustained VT during follow-up. In each case, the VT morphology was different from that in the preoperative or intraoperative EP tests. One of these patients received an ICD and the other has been successfully treated with amiodarone and mexiletene. Six patients who did not have inducible VT 1 week postoperatively have died since discharge. Two had sudden deaths attributed to ventricular fibrillation 7 and 18 months postoperatively. Two patients died of complications of congestive heart failure at 1 and 36 months of follow-up.
Among the remaining patients, one died of a myocardial infarction at 8 months of follow-up and the other died of sepsis at 6 months of follow-up.

\section{DISCUSSION}

Long-term results. In this series there was a perioperative mortality of $15 \%$, which is similar to the results of others, ${ }^{2,6-8}$ including the findings of a multicenter registry. ${ }^{12}$ Eighty-six percent of the remaining patients underwent successful ER and no significant difference in success rate could be discerned between patients who underwent a map-guided ER and those who underwent a visually-directed resection. Although all patients had inducible, sustained VT preoperatively, VT was inducible intraoperatively in only 22 of the 33 patients (67\%). No morphologic characteristic of the VT was associated with the out- 
come of surgery. Concordance of VT morphologies preoperatively and intraoperatively was present in only $43 \%$ of patients and was not significantly associated with operative success or failure in eradicating VT. However, patients who had multiple intraoperative VT morphologies had a higher rate of operative success than the patients who had a single intraoperative VT morphology.

Intraoperative induction of VT. The finding that intraoperative success in inducing VT was only $67 \%$ in this study was surprising in that the programmed stimulation protocol was identical to that used preoperatively. Conceivably, the use of anesthetic agents may have influenced the inducibility of VT in the operating room, although the agent used (fentanyl) produces few electrophysiologic effects. ${ }^{9}$

The issue of preoperative and intraoperative concordance of VT morphologies has not been addressed previously. We found that $\mathrm{VT}$ concordance was present in less than half of our patients and that it was not predictive of surgical outcome. Therefore although the morphology of VT induced in the operating room may be different from the morphology of the VT induced preoperatively, the outcome of ER does not appear to be adversely affected by this difference. Factors that may have accounted for the absence of VT concordance preoperatively and intraoperatively include the effects of anesthetic agents, cardiopulmonary bypass, and ventriculotomy.

Multiple VT morphologies. Miller et al. ${ }^{4}$ reported that certain morphologic variables are predictive of failure in ER, including multiple spontaneous (but not induced) morphologies, disparate sites of VT morphologies, right bundle branch block (RBBB) morphology, and certain locations of VT foci (inferior wall, papillary muscles, and septum). Others ${ }^{10,11}$ found no significant effect of multiple or single VT morphologies, mapping, or inferior wall origin on surgical success. In the present study, the presence of multiple VT morphologies intraoperatively correlated with a higher surgical success rate. It may be that the higher success rate in patients who have multiple VT morphologies is attributable to a more widespread ER than in patients who had only one VT morphology.

Long-term follow-up. We found that two patients who did not have inducible VT 1 week postoperatively experienced recurrences of VT at a later time. Of note is that in both patients the morphology was different from that of any of the VTs before ER. The possibility of a false negative 1 week postoperative EP study exists, but it is also possible that the patients developed recurrent VT because of a new
VT focus that was not present at the time of the operation. Additionally, two patients who also did not have inducible VT postoperatively experienced VF arrests and died in the follow-up period, along with three others who died of cardiac causes and another who died of sepsis.

Map-guided versus visually-directed ER. Map-directed FR and visually-directed $\mathrm{ER}$ have been reported to have comparable success rates. This was also the case in the present study. However, in the present study, visually-directed ER was performed only in patients in whom VT was not inducible in the operating room. A randomized comparison of the two techniques will be needed to determine if their success rates are significantly different.

Clinical implications. In conclusion, the outcome of $\mathrm{ER}$ is not adversely affected by discordance between the morphologies of VTs induced in the EP laboratory and in the operating room. However, every effort should be made to induce as many different types of monomorphic VT as possible, because the surgical success rate may be lower if only one type of VT is induced intraoperatively than if multiple morphologies of VT are induced.

We thank Marion Maguire for her secretarial assistance.

\section{REFERENCES}

1. Couch OA. Cardiac aneurysm with ventricular tachycardia and subsequent excision of aneurysm. Circulation 1959; 20:251-3.

2. Mason JW, Stinson EB, Winkle RA, Oyer PE, Griffen JC, Ross DL. Relative efficacy of blind ventricular aneurysm resection for the treatment of recurrent ventricular tachycardia. Am J Cardiol 1982;49:241-50.

3. Krafchek J, Lawrie GM, Roberts R, Magro SA, Wyndham CRC. Surgical ablation of ventricular tachycardia: improved results with a map-directed regional approach. Circulation 1986;73:1239-47.

4. Miller JM, Kienzle MG, Harken AH, Josephson ME. Mor phologically distinct sustained ventricular tachycardias in coronary artery disease: significance and surgical results. J Am Coll Cardiol 1984;4:1073-9.

5. Gallagher JJ, Kasell JH, Cox JL, Smith WM, Ideker RE, Smith WM. Techniques of intraoperative electrophysiologic mapping. Am J Cardiol 1982;49:221-40.

6. Zee-Cheng CS, Kouchoukos NT, Connors JP, Ruffy R. Treatment of life-threatening ventricular arrhythmias with nonguided surgery supported by electrophysiologic testing and drug therapy. J Am Coll Cardiol 1989;13:153-62.

7. Ostermyer J, Breithart G, Kolvenbach R, Borgreffe M, Seipal L, Schultze HD, Bircks W. The surgical treatment of ventricular tachyarrhythmias. I Thorac Cardiovase Surg 1982;84:704 15.

8. Haines DE, Lerman BB, Kron IL, DiMarco JD. Surgical ablation of ventricular tachycardia with sequential map-guided subendocardial resection: electrophysiologic assessment and long-term follow-up. Circulation 1988;77:131-41.

9. Hunt GB, Ross DL. Comparison of effects of three anesthetic agents on induction of ventricular tachycardia in a canine 
model of myocardial infarction. Circulation 1988;78:221-6.

10. Brandt B, Martins JB, Kienzle MG. Predictors of failure after endocardial resection for sustained ventricular tachycardia. $J$ Thorac Cardiovasc Surg 1988;95:495-500.

11. Josephson ME, Horowitz LN, Farshidi A, Spielman SR, Michelson EL, Greenspan AM. Recurrent sustained ventric- ular tachycardia. 4. Pleomorphism. Circulation 1979:59:459 68.

12. Ostermeyer J, Kirklin JK, Borgreffe M, Cox JL, Breithardt G Bircks W. Ten years electrophysiolgically guided direct oper ations for malignant ischemic ventricular tachycardia-re sults. Thorac Cardiovasc Surg 1989;37:20-7.

\title{
Irregular ventricular tachycardia: A possible manifestation of longitudinal dissociation within the reentry pathway
}

\begin{abstract}
Sustained monomorphic ventricular tachycardia is usually regular; that is, it is associated with constant R-R intervals. In several cases, however, the cycles of ventricular tachycardia are more or less variable. Fifty-four cases of sustained monomorphic ventricular tachycardia were evaluated in order to assess whether tachycardia was regular. Nine cases were defined as irregular (i.e., the R-R cycles varied by more than $\mathbf{4 0}$ msec throughout a 1-minute recording). In five cases tachycardia was "regularly irregular," since the R-R cycles could be divided into two separate groups: the group of long cycles and that of short cycles. In these cases the variability manifested according to a defined and constant pattern: bigeminal pattern (alternation of short and long cycles), trigeminal pattern (two short cycles followed by a long cycle), and so on. The regular variability of tachycardia cycle length suggests one of the following possibilities. (1) There are two alternative circuits (a short circuit and a long circuit) that share the same exit pathway. Whenever the reciprocating impulse runs through the short circuit, the R-R cycle is short; but if a block in the short circuit occurs, the impulse runs through the long circuit, resulting in a long R-R cycle. (2) There is a longitudinal dissociation within the reentry circuit; two separate pathways with different inherent conduction velocities are present. When the impulse runs through the fast pathway, the R-R cycle is short; whereas when a block in the fast pathway occurs, the impulse traverses the slow pathway, resulting in a long R-R cycle. (AM HEART J 1992;124:1506.)
\end{abstract}

Giuseppe Oreto, MD, Gaetano Satullo, MD, Francesco Luzza, MD, Antonino Donato, MD, Ignazio M. Scimone, MD, and Alfredo Cavalli, MD Messina, Italy

Sustained monomorphic ventricular tachycardia (VT) is usually regular; that is, the $\mathrm{R}-\mathrm{R}$ cycles are constant. Polymorphic and nonsustained VT, in contrast, are often associated with variable $R-R$ intervals. In some instances, however, sustained

From Istituto Pluridisciplinare di Clinica Medica, Cattedra di Malattie Cardiovascolari, Università di Messina, Messina, Italy.

Received for publication Jan. 21, 1992; accepted June 15, 1992.

Reprint requests: Giuseppe Oreto, MD, Via Terranuva, 9, 98122 Messina, Italy.

4/1/41300 monomorphic VT reflects irregular $\mathrm{R}-\mathrm{R}$ intervals. This has been observed particularly before spontaneous termination of VT. ${ }^{1}$ This study is focused on the incidence and the mechanism of $R-R$ cycle variability in sustained VT.

\section{METHODS}

Fifty-four patients with sustained monomorphic VT were selected. All of them had had a previous myocardial infarction and had been admitted to the hospital for treatment of an arrhythmia. VT was diagnosed on the basis of results of a surface ECG according to current criteria. ${ }^{2-4}$ For each patient, a 12-lead ECG was available, as well as a con- 